\title{
2D magnetic field warp reversal in images taken with DIXI (dilation x-ray imager)
}

S. R. Nagel, T. J. Hilsabeck, M. J. Ayers, B. Felker, K. W. Piston, T. Chung, P. M. Bell, D. K. Bradley, G. W. Collins, J. D. Kilkenny, J. D. Hares, A. K. L. Dymoke-Bradshaw

August 14, 2013

SPIE Optics and Photonics - Target Diagnostics Physics and Engineering for Inertial Confinement Fusion II San Diego, CA, United States August 25, 2013 through August 29, 2013 
This document was prepared as an account of work sponsored by an agency of the United States government. Neither the United States government nor Lawrence Livermore National Security, LLC, nor any of their employees makes any warranty, expressed or implied, or assumes any legal liability or responsibility for the accuracy, completeness, or usefulness of any information, apparatus, product, or process disclosed, or represents that its use would not infringe privately owned rights. Reference herein to any specific commercial product, process, or service by trade name, trademark, manufacturer, or otherwise does not necessarily constitute or imply its endorsement, recommendation, or favoring by the United States government or Lawrence Livermore National Security, LLC. The views and opinions of authors expressed herein do not necessarily state or reflect those of the United States government or Lawrence Livermore National Security, LLC, and shall not be used for advertising or product endorsement purposes. 


\title{
2D magnetic field warp reversal in images taken with DIXI (dilation x-ray imager)
}

\author{
Sabrina R. Nagel ${ }^{a}$, T.J. Hilsabeck ${ }^{b}$ M.J. Ayers ${ }^{a}$, B. Felker ${ }^{a}$, K.W. Piston ${ }^{a}$, T. \\ Chung $^{b}$, P.M. Bell ${ }^{a}$, D.K. Bradley ${ }^{a}$, G.W. Collins ${ }^{a}$, J.D. Kilkenny ${ }^{b}$, J.D. Hares ${ }^{c}$, \\ A.K.L. Dymoke-Bradshaw ${ }^{c}$ \\ ${ }^{a}$ Lawrence Livermore National Laboratory, 7000 East Avenue, Livermore CA, USA; \\ ${ }^{b}$ General Atomics, 3550 General Atomics Court, San Diego CA, USA; \\ ${ }^{c}$ Kentech Instruments Ltd., Howbery Park, Wallingford, Oxfordshire, UK;
}

\begin{abstract}
DIXI utilizes pulse-dilation technology ${ }^{1}$ to achieve x-ray imaging with temporal gate times below 10 ps. $^{2}$ The longitudinal magnetic field used to guide the electrons during the dilation process results in a warped image, similar to an optical distortion from a lens. Since the front end, where $\mathrm{x}$-rays are converted into electrons at the beginning of the magnetic field, determines the temporal resolution these distortions influence the temporal width of the images at the back end, where it is captured. Here we discuss the measurements and methods used to reverse the magnetic warp effect in the DIXI data. The x-ray measurements were conducted using the COMET laser facility at the Lawrence Livermore National Laboratory.
\end{abstract}

Keywords: gated, x-ray, imager, time dilation, de-warp, magnetic field

\section{INTRODUCTION}

The performance of inertial confinement fusion (ICF) targets relies on the symmetric implosion of DT fuel in order to form a uniform central hot spot with high enough areal density and temperature to achieve ignition. ${ }^{4}$ Gated broadband $\mathrm{x}$-ray imaging at energies exceeding $8 \mathrm{keV}$ is used to diagnose temporal and spatial histories of the implosion symmetry and hot spot non-uniformities. These framing cameras operate using a voltage impulse to gate individual frames and can currently achieve temporal resolutions of 40 to $100 \mathrm{ps}^{5}{ }^{5}$ Simulations predict features around bang time, which can only be resolved by faster gated imagers. ${ }^{3}$ To optimize the target performance and validate code predicitions it is important to diagnose those interactions at a higher frame rate.

DIXI (DIlation X-ray Imager), a new x-ray framing camera, was designed to work at shot neutron yields of up to $10^{17}$. Characterization measurements for spatial and temporal resolution of DIXI have been presented in references 1-3. The working principle of DIXI is briefly described in section 2. The diagnostic uses pulse-dilation of an electron signal to achieve temporal gate times of $<10$ ps. ${ }^{1}$ Possible uses for this technology include: measuring high energy electron transport rates in fast ignition experiments, analyzing the symmetry of late stage implosion of high energy targets and investigating burn wave dynamics for igniting targets.

Further author information: (Send correspondence to S.R. Nagel)

S.R. Nagel: E-mail: nagel7@llnl.gov, Telephone: 19254227739 


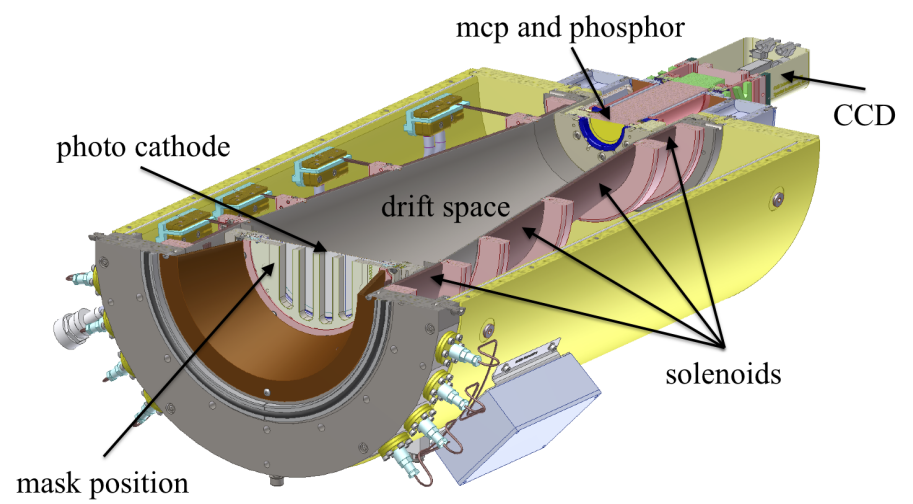

Figure 1: Sketch of the bottom half of DIXI showing the four photo cathode strips, $50 \mathrm{~cm}$ long drift space, 4 solenoids and the back end including MCP, phosphor and CCD.

The pulse dilation principle uses an electron signal traveling in an evacuated drift space. A longitudinal magnetic field is used to guide the electron signal in the DIXI drift space. This magnetic field is non-uniform in order to de-magnify a $12 \mathrm{~cm}$ photo cathode image onto a $4 \mathrm{~cm}$ micro-channel plate. This non-uniformity results in a warped image, similar to an optical distortion from a lens. Since the front end, where the drift electrons are generated, determines the temporal resolution, the image distortions due to the magnetic field influence the temporal width of the images at the back end. Here we discuss how we obtain the correction maps applied to the image to un-distort or de-warp it.

\section{DIXI WORKING PRINCIPLE}

DIXI uses pulse dilation of an electron signal from a transmission photo cathode (PC) to achieve short temporal gate times of $<10 \mathrm{ps}$, much less than conventional framing cameras that use a voltage impulse to gate individual frames $(40-100 \mathrm{ps})$. Figure 1 shows a diagram of DIXI indicating the main sections. Figure 2 illustrates the working principle of the instrument. An electron signal is

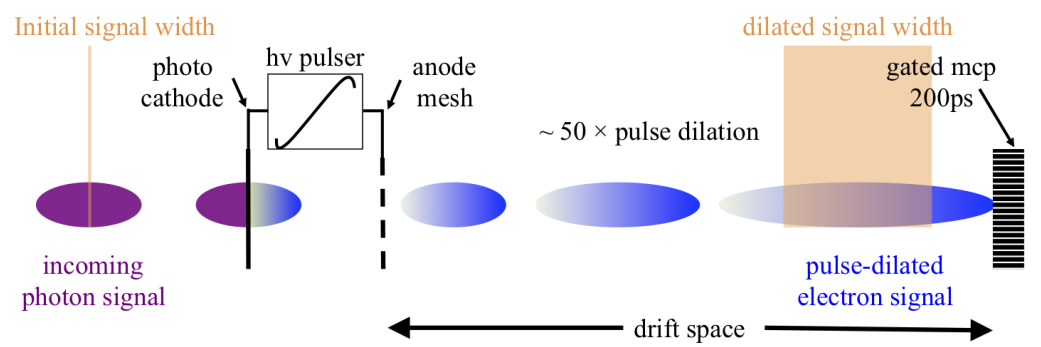

Figure 2: Illustration of the DIXI working principle. X-rays are converted into electrons at the photocathode $\rightarrow$ accelerated by a time varying electric field $\rightarrow$ energy dispersion $\rightarrow$ signal stretches as it traverses the drift region $\rightarrow$ sampled by a gated MCP. X-rays are illustrated in purple and electrons in blue. The energy spread of the electrons is indicated by the color gradient. 


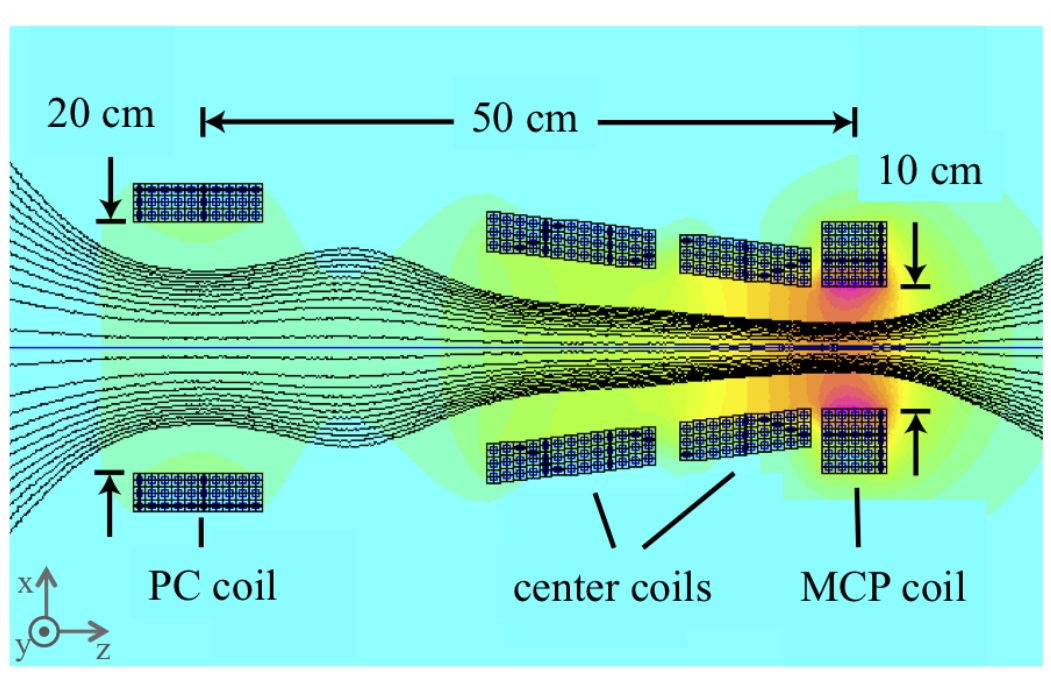

$5.900 \mathrm{e}-001:>6.211 \mathrm{e}-001$ $5.590 \mathrm{e}-001: 5.900 \mathrm{e}-001$

$5.279 e-001: 5.590 e-001$

$4.969 \mathrm{e}-001: 5.279 \mathrm{e}-001$

$4.658 \mathrm{e}-001: 4.969 \mathrm{e}-001$

$4.348 \mathrm{e}-001: 4.658 \mathrm{e}-001$

$4.037 \mathrm{e}-001: 4.348 \mathrm{e}-001$

$3.727 \mathrm{e}-001: 4.037 \mathrm{e}-001$

$3.416 \mathrm{e}-001: 3.727 \mathrm{e}-001$

$3.106 \mathrm{e}-001: 3.416 \mathrm{e}-001$

$2.795 \mathrm{e}-001: 3.106 \mathrm{e}-001$

$2.485 e-001: 2.795 e-001$

$2.174 \mathrm{e}-001: 2.485 \mathrm{e}-001$

$1.864 \mathrm{e}-001: 2.174 \mathrm{e}-001$

$1.553 \mathrm{e}-001: 1.864 \mathrm{e}-001$

$1.243 \mathrm{e}-001: 1.553 \mathrm{e}-001$

$9.323 e-002: 1.243 e-001$

$6.218 \mathrm{e}-002: 9.323 \mathrm{e}-002$

$3.113 e-002: 6.218 \mathrm{e}-002$

$<8.098 \mathrm{e}-005: 3.113 \mathrm{e}-002$

Density Plot: |B|, Tesla

Figure 3: Illustration of the modeled cylindrically symmetric magnetic field map for coil currents of: 142 A, 150 A, 200 A, 1000 A (from the front of the instrument to the back). Magnetic field lines are indicated by the black lines.

generated as x-rays hit the $\mathrm{Au}$ or $\mathrm{CsI}$ transmission $\mathrm{PC}^{6}$ of the framing camera. The $\mathrm{PC}$ is pulsed and the electrons are accelerated by a time varying electric field, which leads to an energy dispersion of the electron signal. Electrons generated earlier in time have a higher velocity than electrons generated later. The electrons pass through a drift region in which their velocity distribution leads to a dilation or stretching of the electron signal. To keep the electrons from defocusing in the drift tube, a longitudinal magnetic field is applied, which also de-magnifies the image from the PC onto the micro-channel plate $(\mathrm{MCP})$ by $\approx 2.5 \times$. The dilated signal then hits the gated MCP detector, which is followed by a phosphor screen, fiber block and CCD or film pack. The MCP pulse is timed relative to the PC pulse to gate the electron signal. More detailed descriptions of the working principle as well as the temporal and spatial resolution of the instrument can be found in references 1-3. In this paper we focus on the effect of the magnetic field on the electrons.

\section{THE MAGNETIC FIELD}

The longitudinal magnetic field used for electron guiding is generated by four coils. Each coil has an independent voltage to which the capacitors can be charged. The magnetic field is then induced by the current through the coils, generated when the capacitors are discharged. The rise time of the DIXI magnetic field is $\approx 500 \mu \mathrm{s}$.

\subsection{Magnetic field pincushinoning}

An illustration of the magnetic field strength within the drift space can be seen in figure 3 . The figure also shows magnetic field lines in black. The four solenoids, one PC coil, two center coils and one MCP coil, are indicated by the dark rectangular shapes. The PC is positioned in the center 


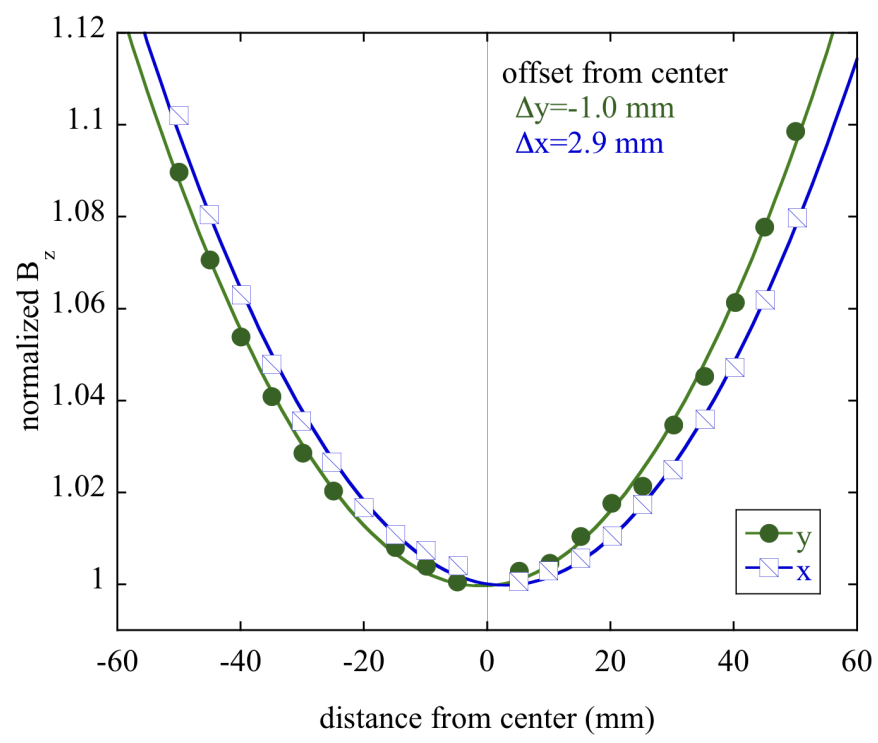

Figure 4: Magnetic field profile across the PC in left-right (x) and up-down (y) direction.

of the PC coil and the MCP in the center of the MCP coil. The magnetic field at the PC plane is orthogonal to the PC surface.

The measured magnetic field profile across the $\mathrm{PC}$ in the $\mathrm{x}$ and $\mathrm{y}$ directions can be seen in figure 4. The small offset of the peak magnetic field from the centers is within the error of the measurement and does not significantly affect the center of the image recorded on the MCP.

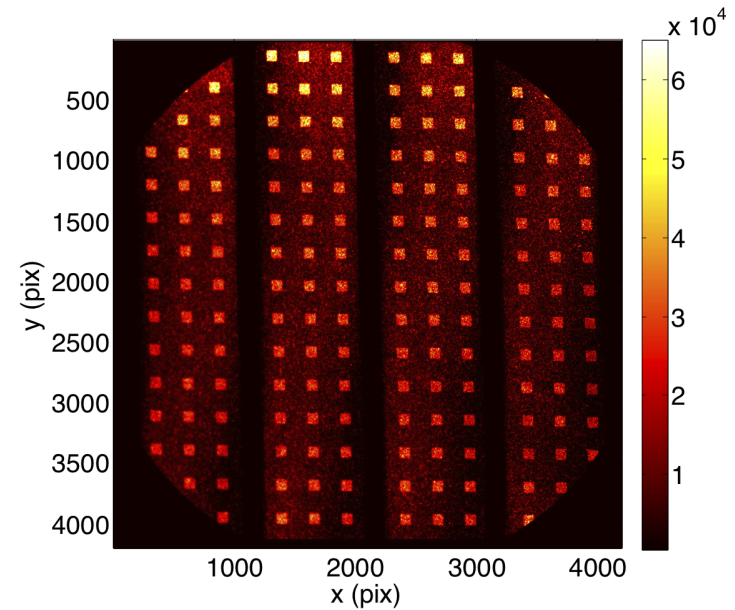

Figure 5: Original "DC" image with mask in front of PC.

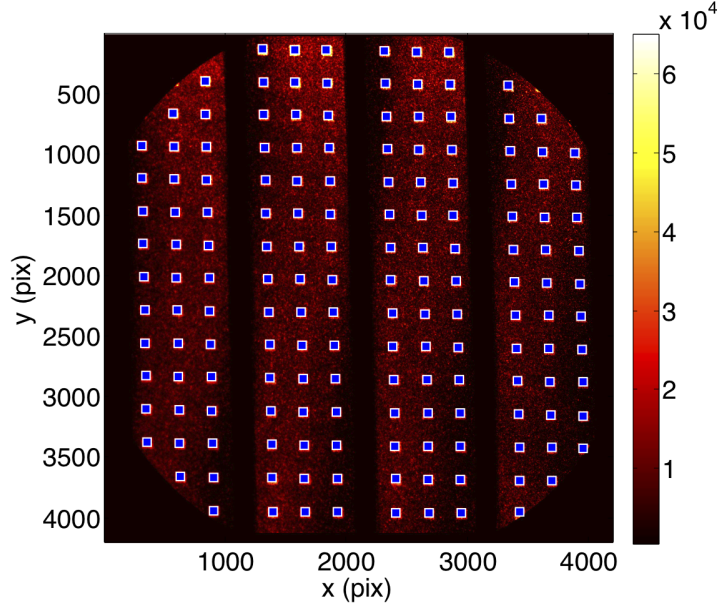

Figure 6: Original image with found centers shown in blue squares with white frame. 


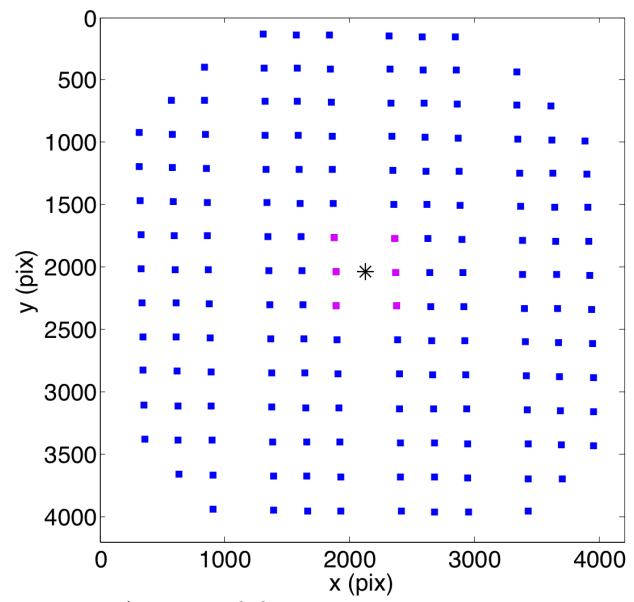

Figure 7: Array of found centers with the positions used to find the center, indicated by the star, in magenta.

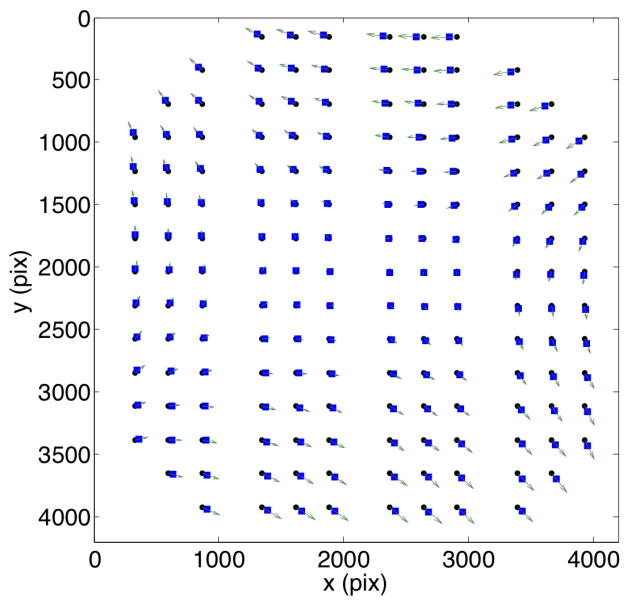

Figure 8: 2D vector plot showing the direction and amplitude of warp for the positions of the transmission centers.

\subsection{Setup and mask specifications}

In order to relate particular points on the $\mathrm{PC}$ to the recorded data at the back end, a mask is placed in front of the $\mathrm{PC}$ to act as a spatial reference. The mask is $250 \mu \mathrm{m}$ thick Tantalum, positioned $\approx$ $1 \mathrm{~cm}$ in front of the PC. It has $2 \mathrm{~mm} \times 2 \mathrm{~mm}$ square holes that are $6 \mathrm{~mm}$ apart and horizontally centered on each strip and a pattern of three squares across each strip, as can be seen in figure 5 . The measurements discussed here were taken with the COMET short pulse laser at the JLF. ${ }^{3}$ Here the short pulse, $1 \mu \mathrm{m}$ laser is focused onto a $50 \mu \mathrm{m}$ thick $\mathrm{Cu}$ foil, and the resulting x-ray impulse is sent directly onto the PC, without the use of imaging pinholes.

When pulsed, the ramp at the PC acts like a traveling shutter along the PC strip and the resulting temporal resolution is $<10 \mathrm{ps}$. As the $\mathrm{x}$-ray impulse created by the COMET short pulse

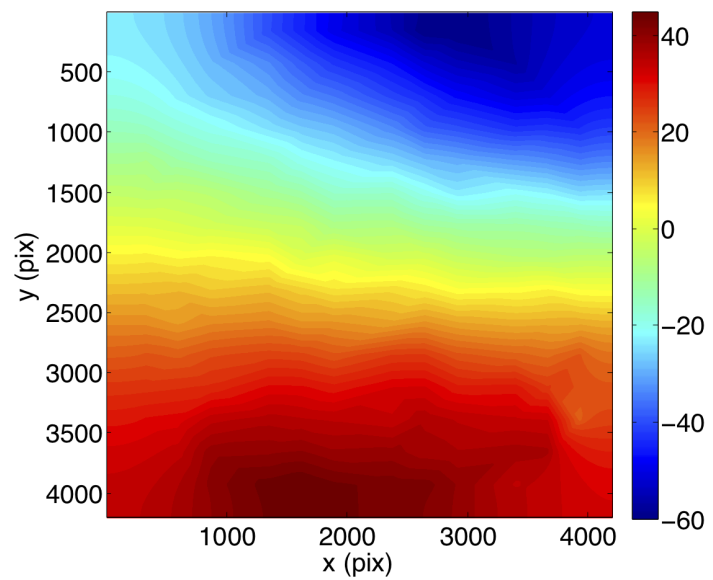

Figure 9: Dewarp map of dx values.

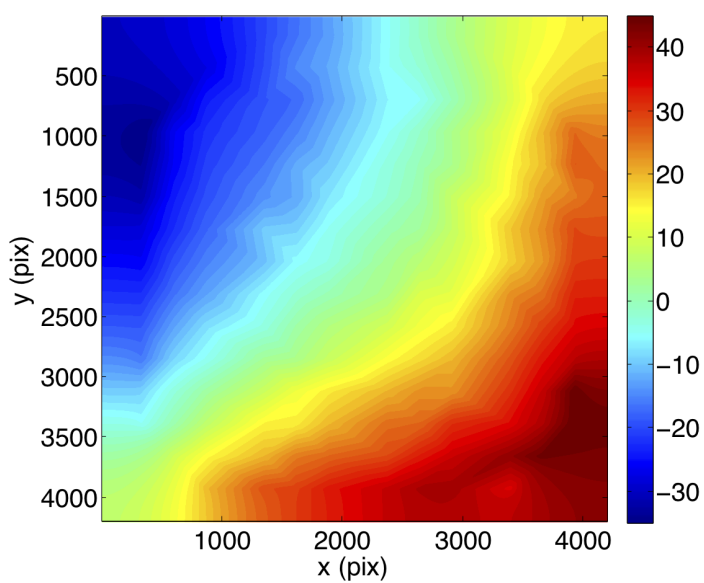

Figure 10: Dewarp map of dy values. 


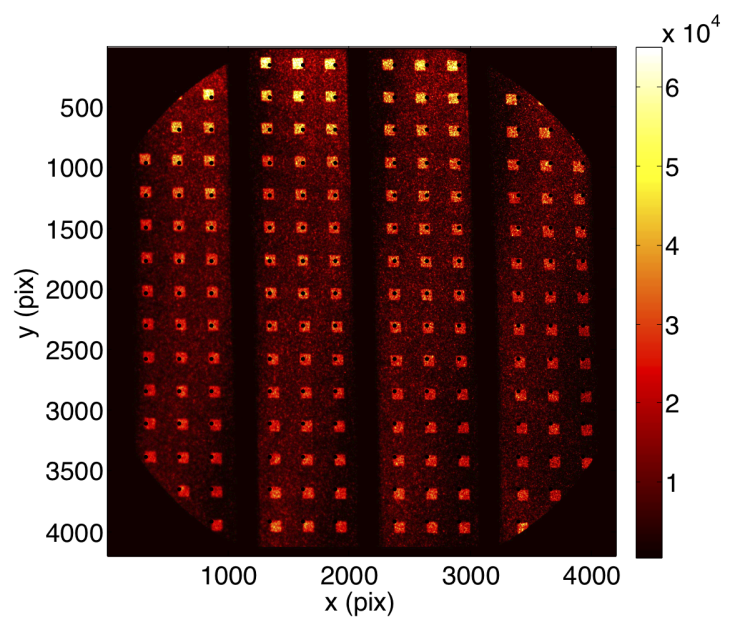

Figure 11: Original "DC" image with mask and overlaid "straight" positions of the centers (black dots).

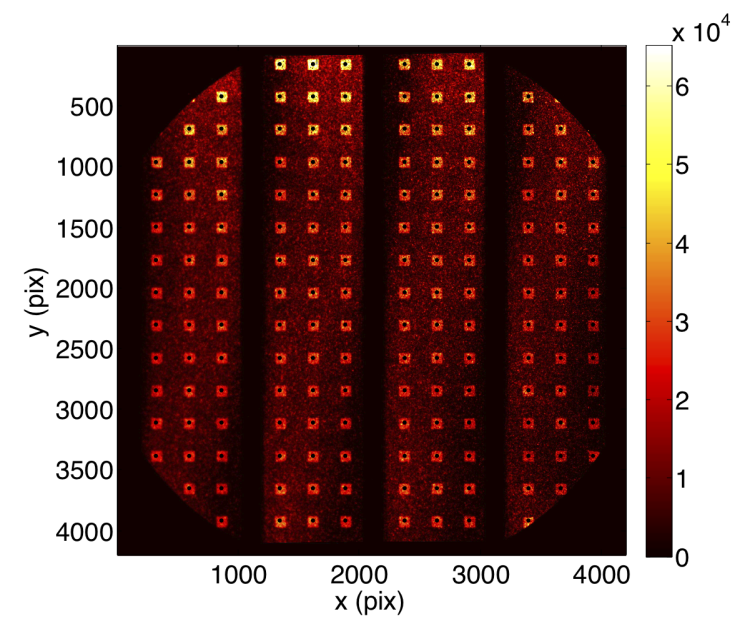

Figure 12: De-warped "DC" image with mask and overlaid "straight" positions of the centers (black dots).

laser is very short $(\leq 5 \mathrm{ps})$, This would only illuminate a small portion of the strip. In order to illuminate the whole PC with the short x-ray impulse the PC is run DC for the magnetic field measurements, i.e. with only a bias applied and not pulsed. However the MCP is pulsed to only investigate the effect of the magnetic field during the relevant time of gating. The instrument is timed so that the MCP gate is optimized for the time of the x-ray impulse reaching the PC and the $\mathrm{PC}$ bias voltage.

\subsection{Magnetic field maps and de-warp}

An image using the mask in front of the PC is shown in figure 5. The four PC strips are clearly visible, as are the mask apertures (small squares). To determine the correction maps for correcting the effect of the magnetic field, an algorithm is used to identify the individual apertures (Figure 6 blue squares) and their center positions as also shown in figure 7 . The six points indicated in magenta are closest to the center of the array and are used to determine the middle point, indicated by the star, and magnification of the array. Using this magnification and the position of the middle point as a fixed center, we obtain an array of positions where the centers should fall without any magnetic field distortions. This is plotted as the black dots in figure 8. Comparing these two arrays gives the differences in position for the $\mathrm{x}$ and $\mathrm{y}$ directions, indicated by the arrows in figure 8 . Note that the length of the arrows in figure 8 is exaggerated to enhance their visibility. These shifts are used for two triangle based linear interpolations, one in $\mathrm{x}$ and one in $\mathrm{y}$, from which we obtain two maps $\mathrm{dx}$ and $\mathrm{dy}$, shown in figures 9 and 10, respectively. The maps give the values of how much each initial pixel has to be moved in $\mathrm{x}$ and $\mathrm{y}$ to obtain a de-warped image. This is achieved by mapping each pixel onto a new image while shifting the position of the pixel in the new image by the amount specified in the two maps.

Figure 10 shows that in the temporal dimension, dy, the correction is up to 50 pixel. This corresponds to $4 \mathrm{ps}$, and would in itself not be a significant amount, however the whole difference across the image of 85 pixel is closer to $7 \mathrm{ps}$, which is just above the possible minimum gate width of $\approx 5$ ps. 


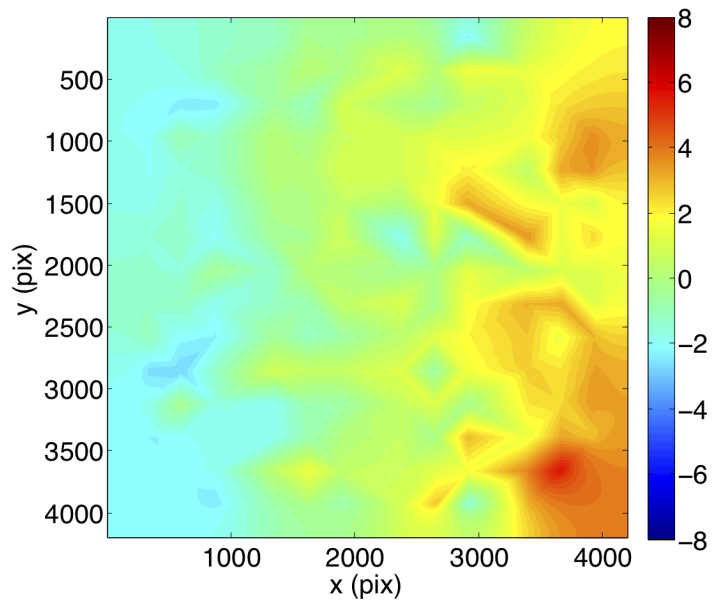

Figure 13: 2nd de-warp map of dx values from $1 \times$ corrected image.

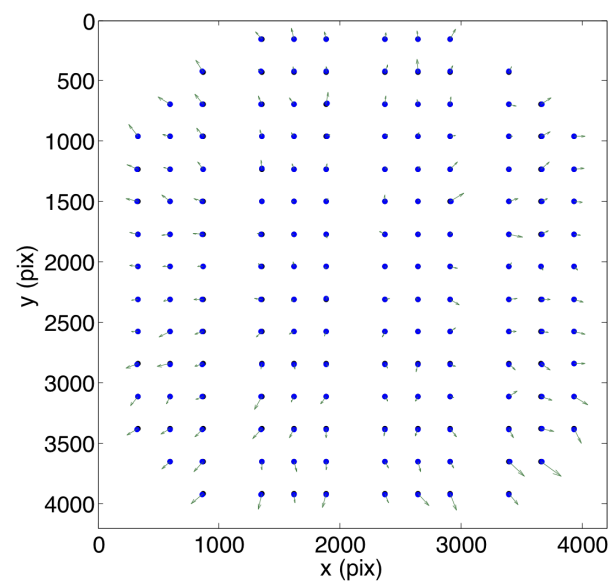

Figure 15: 2D vector plot of the transmission centers after the de-warp algorithm has been applied once.

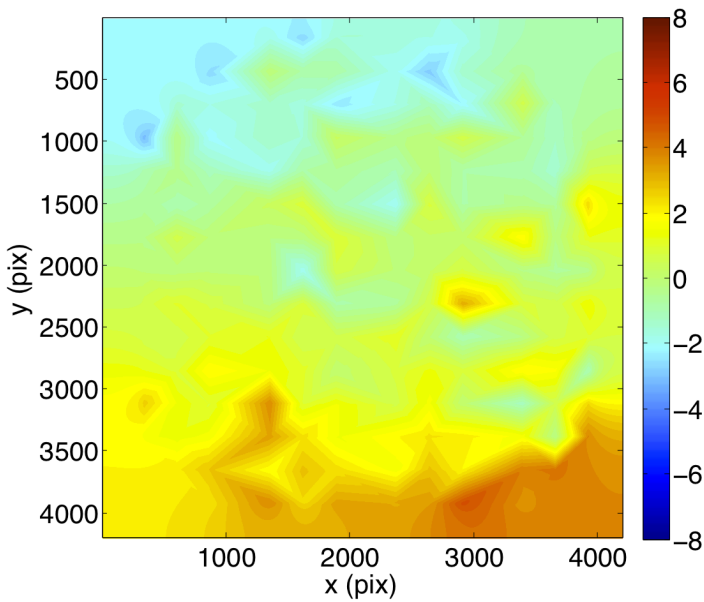

Figure 14: 2nd de-warp map of dy values from $1 \times$ corrected image.

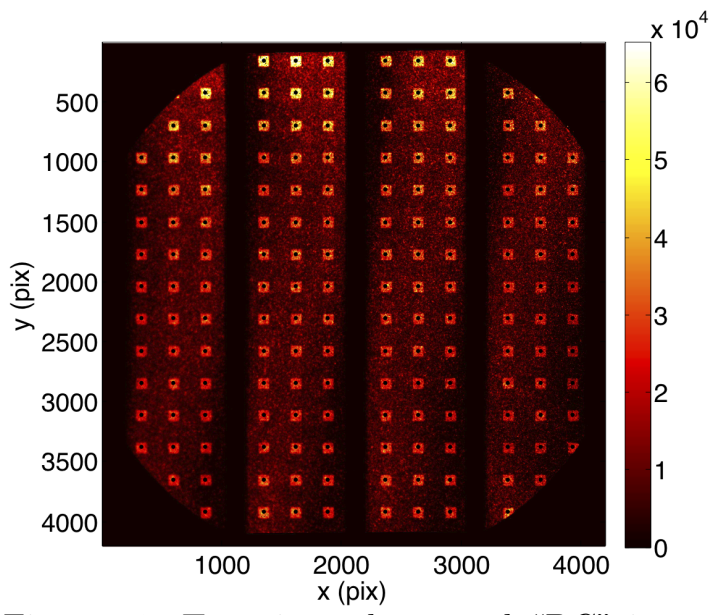

Figure 16: Two times de-warped "DC" image with mask and overlaid "straight" positions of the 2nd time found centers (black dots).

An image with the original image overlaid with the "straight" positions of the centers can be seen in figure 11. Figure 12 shows the image after the de-warp is applied with the same centers as in figure 11. The improvement from this single 2D de-warp is unmistakable.

To test whether the algorithm has converged to a solution, the image correction is re-applied to the $1 \times$ de-warped image. This gives a second pair of de-warp maps, see figures 13 and 14 . The residual error is small here with a total shift of $\leq 10$ pix which corresponds to $\leq 0.8$ ps. A $2 \mathrm{D}$ vector plot for the 2 nd application and the $2 \times$ de-warped image with the 2 nd found array can be seen in figures 15 and 16, respectively. Due to the small correction for the second de-warp a single correction is sufficient for data analysis. 


\section{SUMMARY}

In summary we have shown that the magnetic field effect resulting in a "warped" or distorted image can be corrected by remapping the image using two de-warp maps, one each for the $\mathrm{x}$ and $\mathrm{y}$ direction, respectively. We determined that due to the 1st iteration of the de-warp correction, the shift for a 2nd iteration becomes smaller than 1 ps and one iteration is considered sufficient. These maps are unique for each DIXI instrument and change for different magnetic field settings.

\section{ACKNOWLEDGMENTS}

The Authors would like to acknowledge the support of the staff at the Jupiter Laser Facility. Lawrence Livermore National Laboratory is operated by Lawrence Livermore National Security, LLC, for the U.S. Department of Energy, National Nuclear Security Administration under Contract No. DE-AC52-07NA27344.

(LLNL-CONF-?????)

\section{REFERENCES}

1. Hilsabeck,T. J., Hares, J. D., Kilkenny, J. D., Bell, P. M., Dymoke-Bradshaw, A. K. L., Koch, J. A., Celliers, P. M., Bradley, D. K., McCarville, T., Pivovaroff, M., Soufli, R. and Bionta, R., "Pulse-dilation enhanced gated optical imager with 5 ps resolution," Rev. Sci. Instrum., 81, 10E317, (2010).

2. Nagel, S .R., Ayers, M. J., Felker, B., Hilsabeck, T. J., Chung, T., Smith, R. F., Bell, P. M., Bradley, D. K., Collins, G. W., Kilkenny, J. D., Sammuli, B., Hares, J. D. and DymokeBradshaw, A. K. L., "Perforemance measurements of DIXI (dilation x-ray imager) photocathode using a laser produced x-ray source," Proc of SPIE, 8505, 85050H-2 (2012).

3. Nagel, S .R., Hilsabeck, T. J., Bell, P. M., Bradley, D. K., Ayers, M. J., Barrios, M. A., Felker, B., Smith, R. F., Collins, G. W., Jones, O. S., Kilkenny, J. D., Chung, T., Piston, K., Raman, K. S., Sammuli, B., Hares, J. D. and Dymoke-Bradshaw, A. K. L., "Dilation x-ray imager a new/faster gated x-ray imager for the NIF," Rev. of Sci. Instrum., 83, 10E116 (2012); http://dx.doi.org/10.1063/1.4732849.

4. S. Atzeni and J. Meyer-ter Vehn, "The Physics of Inertial Fusion," International Series of Monographs on Physics (Clarendon Press, Oxford, 2004); J. D. Lindl, "Inertial Confinement Fusion: The Quest for Ignition and Energy Gain Using Indirect Drive" (Springer-Verlag, New York, 1998).

5. Bradley, D. K., Bell, P. M., Landen, O. L., Kilkenny, J. D. and Oertel, J., "Development and characterization of a pair of 30-40 ps x-ray framing cameras," Rev. Sci. Instrum., 66, 716 (1995).

6. Henke, B. L., Knauer, J. P. and Premaratne, K., "The charaterisation of x-ray photocathodes in the $0.110 \mathrm{keV}$ photon energy region," J. Appl. Phys., 52(3), 1509 (1981). 